\title{
April 2019 Critical Care Case of the Month: A Severe Drinking Problem
}

Francisco J. Marquez II MD

\author{
Department of Pulmonary and Critical Care Medicine \\ Banner University Medical Center/University of Arizona - Phoenix \\ Phoenix, AZ USA
}

\section{History of Present IIIness}

A 55-year-old Caucasian man, presented to an outside hospital with altered mental status.

\section{Past Medical/Social History}

- Severe alcohol and intermittent fentanyl abuse

- Homelessness

\section{Physical Exam}

- Hypothermic and hypertensive.

- Patient encephalopathic without any acute deficits

- Pupils are normal sized and react to light

Which of the following should be obtained or done in his initial evaluation?
1. $\mathrm{CBC}$
2. Electrolytes
3. Give naloxone (Narcan $\AA)$ and glucose
4. 1 and 3
5. All of the above 


\section{Correct! \\ 5. All of the above}

The differential diagnosis for an altered mental status is quite large. A popular mnemonic that lists many of the possible causes of altered mental state is AEIOU TIPS (1). Each letter stands for a possible cause of an altered mental status (Table 1).

Table 1. Causes of an altered mental status.

A - Alcohol/Acidosis.

E - Endocrine/Epilepsy/Electrolytes/Encephalopathy.

I - Infection- meningitis, encephalitis, sepsis, septic shock; pneumonia, urinary tract infection, occult osteomyelitis.

O - Opiates, Overdose.

U - Uremia/Underdose.

T - Trauma - head injury, blood loss (shock).

I - Insulin.

Because the differential is so large, a generalized workup is initially indicated. This includes a CBC and electrolytes and a UA but would also include a urinalysis (UA), blood sugar and arterial blood gas. It is common to give naloxone and glucose on initial evaluation because narcotic overdose and hypoglycemia are so common.

The patient's $\mathrm{CBC}$ is unremarkable. However, the electrolytes are $\mathrm{Na}^{+} 147 \mathrm{mEq}, \mathrm{K}^{+} 5.0$ $\mathrm{mEq}$, bicarbonate $9 \mathrm{mEq}$, and $\mathrm{Cl}^{-113} \mathrm{mEq}$.

\section{The electrolytes reveal?}

1. A high anion gap metabolic acidosis

2. A metabolic alkalosis

3. A normal anion gap metabolic acidosis

4. 1 and 3

5. All of the above 


\section{Correct! \\ 1. A high anion gap metabolic acidosis}

The patient has a metabolic acidosis as evidenced by the low serum bicarbonate. Metabolic acidosis can be separated into normal or high anion gap metabolic acidosis (2). A normal anion gap is less than $11 \mathrm{mEq} / \mathrm{L}$ and calculated by the following formula: Anion gap $=\mathrm{Na}-(\mathrm{Cl}+\mathrm{HCO} 3)$.

A normal anion gap acidosis is caused by a loss of bicarbonate usually through the gastrointestinal tract or the kidneys and is often associated with a high chloride. A high anion gap occurs when an acid accumulates. The mnemonic MUDPILES is commonly used to remember the causes of increased anion gap metabolic acidosis (Table 2) (3).

Table 2. Causes of an increased anion gap acidosis.

- M-Methanol

- U - Uremia (chronic kidney failure)

- D - Diabetic ketoacidosis

- P - Paracetamol, Propylene glycol (used as an inactive stabilizer in many medications; historically, the "P" also stood for Paraldehyde, though this substance is not commonly used today)

- I - Infection, Iron, Isoniazid (which can cause lactic acidosis in overdose), Inborn errors of metabolism (an especially important consideration in pediatric patients)

- L - Lactic acidosis

- E - Ethylene glycol (Note: Ethanol is sometimes included in this mnemonic as well, although the acidosis caused by ethanol is actually primarily due to the increased production of lactic acid found in such intoxication.)

- S-Salicylates

Our patient's anion gap is elevated $[147-(113+9)]=25 \mathrm{mEq}$. Other laboratory work includes:

- $\mathrm{ABGs}-\mathrm{PaO} 275, \mathrm{PaCO} 2$ 37, $\mathrm{pH} 6.92$

- Serum glucose - $138 \mathrm{mg} / \mathrm{dL}$

- Blood urea nitrogen $-15 \mathrm{mg} / \mathrm{dL}$

- Creatinine $1.8 \mathrm{mg} / \mathrm{dL}$

The patient is becoming increasingly obtunded.

What should be done at this time?

1. Intubate the patient for airway protection

2. Obtain a head CT scan

3. Obtain a drug screen

4. 1 and 3

5. All of the above 


\section{Correct! \\ 5. All of the above}

The immediate need is to support the patient and search for the cause of the acidosis which likely is leading his obtundation. He needs intubation to protect him from aspiration and a drug screen is indicated. In the absence of a focal neurological sign a head CT scan might be controversial but most physicians would probably obtain one. His head CT was reported as unremarkable. He becomes progressively hypertensive with systolic blood pressures into 200's and a nicardipine drip is begun. At this time, he was transferred to Banner University Medical Center - Phoenix.

At arrival pertinent findings on his physical examination include

- An obtunded man with a normal blood pressure

- Pupils equal, round and reactive to light at $3 \mathrm{~mm}$

- No clonus

His ethanol/aspirin/acetaminophen/lithium drug screen was negative.

Which of the following should be done at this time?

1. Begin fomepizole

2. Order an ethylene glycol and methanol level

3. Repeat the urinalysis

4. 1 and 3

5. All of the above 


\section{Correct! \\ 5. All of the above}

Our patient's severe high anion gap metabolic acidosis and obtundation without another apparent cause suggest toxic alcohol ingestion (4). Unfortunately, a suppositional diagnosis needs to be made before confirmation since it normally takes several days before serum levels return. In addition to ethanol the toxic alcohols include ethylene glycol (EG), methanol, and isopropanol. These alcohols exert most of their toxicity by conversion to metabolites through alcohol dehydrogenase. Fomepizole is an inhibitor of alcohol dehydrogenase that should be started when toxic alcohol ingestion is suspected (4). Fomepizole is advantageous because it does not depress the patient's mental status or airway and needs to be administered only every 12 hours. The main drawback of fomepizole is the cost, which can total thousands of dollars. Because this agent is so expensive, clinicians should check its availability at their institution and discuss the plan for use of this antidote, especially for empiric treatment of cases in which the cause of acidosis is unknown.

This patient eventually proved to have ethylene glycol ingestion. Ethylene glycol is the major ingredient of almost all radiator fluid products in the United States. Fluorescein dye is often added to radiator fluid to help mechanics identify the source of a radiator leak. It can be detected in a patient's urine under ultraviolet light several hours after ingestion. In addition, ethylene glycol is metabolized to oxalate and the crystals may be detected in the urine as well as deposited in multiple tissues (4). It is the oxalate that is deposited in the kidney that results in the renal failure that can be caused by ethylene glycol.

Our patient his renal function continued to decline, with a creatinine as high as 6 and he was started on hemodialysis. However, his blood pressure and mental status improved and he was quickly weaned from the nicardipine drip. Four days after transfer he was successfully extubated. Unfortunately, shortly after he was extubated though he his blood pressure rose with systolic readings 200's. He became more somnolent and severely encephalopathic, ultimately requiring re-intubation.

What should be done at this time?

1. Blood cultures for possible sepsis

2. Repeat the electrolytes and arterial blood gases

3. Repeat the head CT scan

4. 1 and 3

5. All of the above 


\section{Correct! \\ 5. All of the above}

Clearly there has been a change resulting in a decline in his mental status. A repeat of the electrolytes, arterial blood gases and the other laboratory values revealed no real change compared to the previous days when he was improving. A repeat head CT scan followed by an MRI showed an acute cortical infarct involving the right parietal, occipital, and posterior temporal lobes (Figure 1).

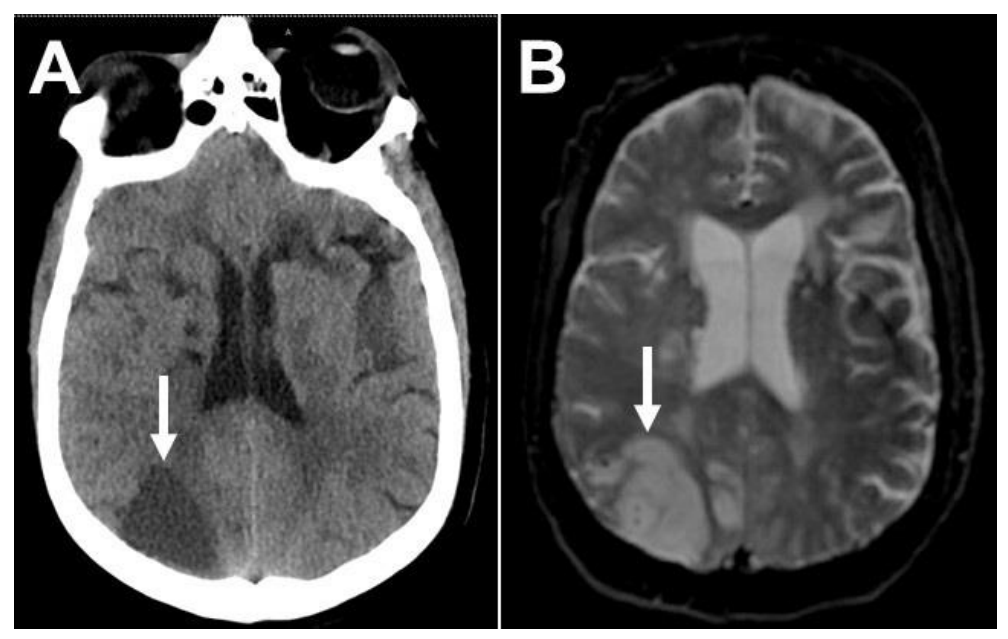

Figure 1. Panel A: Head CT after reintubation and second hypertensive crisis shows an acute cortical infarct involving portions of the right parietal, occipital, and posterior temporal lobes with potential anterior right temporal lobe involvement suggestive of a watershed infarction (arrow). Panel B: Brain MRI confirms the acute cortical infarct in addition to right aspect of pons and left basal ganglia (arrow).

Given the multiple scattered vascular ischemic events in a bilateral distribution and in unusual locations, this presentation would normally be considered embolic in terminal vessel territories. However, the patient did not have any typical vascular embolic phenomena including atrial fibrillation or left ventricular thrombus with a normal TTE. After careful consideration, this case was favored to represent severe ethylene glycol toxicity causing microscopic calcium oxalate crystal emboli (5).

The patient remained on supportive treatment for multiple days, but ultimately improved sufficiently to be transferred to Inpatient Behavioral Health for further treatment of his probable suicide attempt. At the time of transfer, he had residual confusion, memory loss and right full body weakness and atrophy.

\section{References}

1. Emergency Medicine/Altered mental status. Wikibooks. August 30, 2017. Available at: https://en.wikibooks.org/wiki/Emergency Medicine/Altered mental status (accessed 1/28/19). 
2. Anion gap. MDCalc. Available at: https://www.mdcalc.com/anion-gap (accessed $1 / 28 / 19)$.

3. High anion gap metabolic acidosis. Wikipedia. December 7, 2018. Available at: https://en.wikipedia.org/wiki/High anion gap metabolic acidosis (accessed 1/28/19).

4. Keyes DC. Ethylene glycol toxicity. Medscape. December 5, 2017. Available at: https://emedicine.medscape.com/article/814701-overview (accessed 1/28/19).

5. Kruse JA. Methanol and ethylene glycol intoxication. Crit Care Clin. 2012;28:661711. [CrossRef] [PubMed] 